\title{
BU CMi as a Quadruple Doubly Eclipsing System
}

\author{
I. M. Volkov ${ }^{a, b, *}$, A. S. Kravtsova ${ }^{a, b, * *}$, and D. Chochol ${ }^{c, * * *}$ \\ ${ }^{a}$ Lomonosov Moscow State University, Sternberg Astronomical Institute, Moscow, Russia \\ ${ }^{b}$ Institute of Astronomy of the Russian Academy of Sciences, Moscow, Russia \\ ${ }^{c}$ Astronomical Institute of the Slovak Academy of Sciences, Tatranská Lomnica, 05960 Slovak Republic \\ *e-mail: hwp@yandex.ru \\ **e-mail: kravts@yandex.ru \\ ***e-mail: chochol@ta3.sk
}

Received April 21, 2021; revised April 30, 2021; accepted April 30, 2021

\begin{abstract}
We found that the known spectroscopic binary and variable BU CMi $=$ HD65241 $\left(V=6.4-6.7^{\mathrm{m}}\right.$, $\mathrm{A} 0 \mathrm{~V}$ ) is a quadruple doubly eclipsing $2+2$ system. Both eclipsing binaries are detached systems moving in an eccentric orbits: pair "A" with the period $P_{\mathrm{A}}=2.94(e=0.20)$ and pair "B" with the period $P_{\mathrm{B}}=3.26$ $(e=0.22)$. All four components have nearly equal sizes, temperatures and masses in the range $M=3.1-3.4 M_{\odot}$, and A0 spectra. We found the mutual orbit of both pairs around the system barycenter with a period of 6.6 years and eccentricity $e=0.7$. We detected in pairs " $\mathrm{A}$ " and " $\mathrm{B}$ " the fast apsidal motion with the periods $U_{\mathrm{A}}=25.4$ years and $U_{\mathrm{B}}=26.3$ years, respectively. The orbit of each pair shows small nutation like oscillations in periastron longitude. The system is young and it seems that its components does not yet reached the Zero Age Main Sequence (ZAMS). The photometric parallax calculated from the found parameters coincides perfectly with the GAIA DR2 $\pi=0.00407^{\prime \prime} \pm 0.00006^{\prime \prime}$.
\end{abstract}

Keywords: binaries: eclipsing, stars: fundamental parameters, stars: individual (BU CMi), techniques: photometric, techniques: radial velocities

DOI: $10.1134 / \mathrm{S} 1063772921090080$

\section{INTRODUCTION}

The system BU CMi is designated in GCVS as an eclipsing variable EA with the period $P=2.93^{\mathrm{d}}$ and strongly displaced secondary minimum MinIIMinI $=0.37 P[1]$, which means the large eccentricity of the orbit. So, it was included in our program of investigation of the inner structure of the stars [2]. We started the observations in 2012 at Stará Lesná observatory, Slovakia. We managed to fix only one exit from minimum strongly apart of the GCVS ephemeris [1]. Our further observations in the same year did not bring any result, the depth of the only observed eclipse turned out to be insignificant and the system was classified as unpromising for the inner structure investigation. But when the reviews of the bright stars such as MASCARA [3] and TESS [4] became available we returned to analysis of the system. The ASAS data [5] for this bright star were useless as their precision was poor, may be due to overexposure. The first glance to the light curve (LC) built with MASCARA data [3] revealed that there are two groups of eclipses with different but stable periods and displaced secondary minima (see Fig. 1 with more illustrative TESS observations). Using the newly defined ephemeris we have resumed intensive observations of the star at the Simeiz observatory of INASAN with the $60-\mathrm{cm}$ reflector and UBV-photometer constructed by I.M. Volkov [6].

\section{OBSERVATIONS AND DATA REDUCTION}

The log of our observations is presented in Table 1. During observations in 2020 all four minima-two primary and two secondary ones-were recorded for both eclipsing systems observed simultaneously as a single star. HD64963 $(V=8.23, \mathrm{~A} 2)$ at a distance $22^{\prime}$ of the variable served as a standard star for observations using the UBV-photometer with photomultiplier. The observations were carried out according to the standard star-variable star-standard star scheme. The expositions in each of the photometric passbands were equal to 20-30 s, sometimes the background near the standard and variable stars was measured. We used aperture 27.5". The maximum value of the signal from the star did not exceed $200000 \mathrm{~s}^{-1}$ in $B$. The signal was weaker in other photometric passbands. Such rate of the signal gave a correction due to the nonlinearity of the equipment not more than 0 m 005 , which was taken 


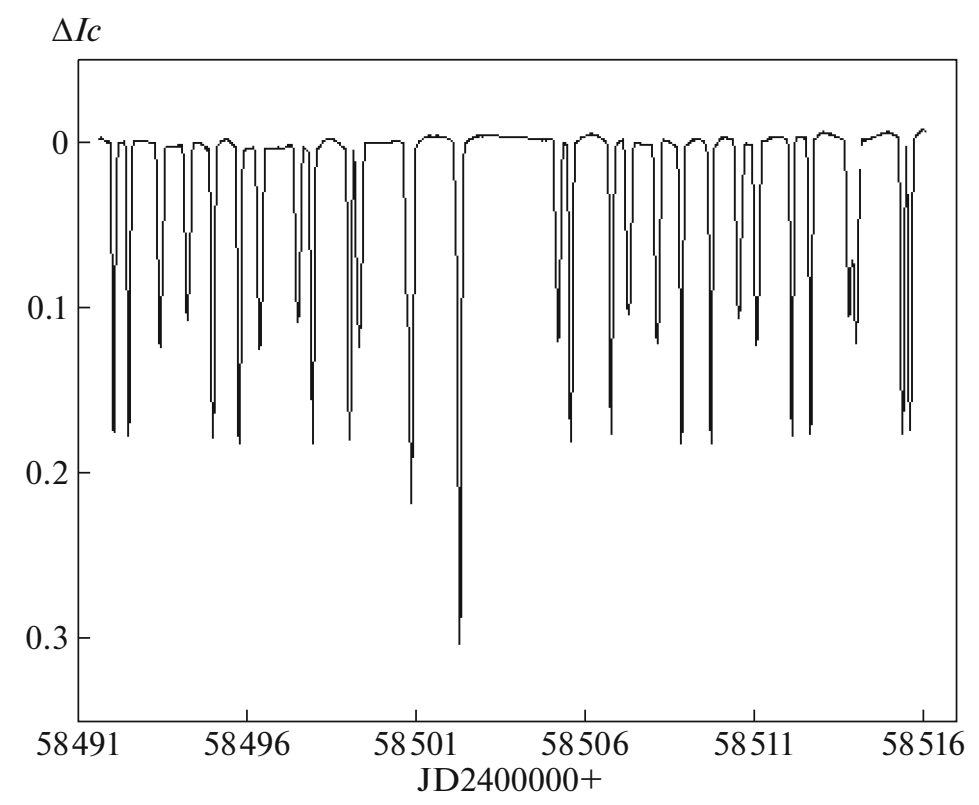

Fig. 1. TESS observations of BU CMi. Two groups of eclipses of stars "A" and "B" with stable periods and displaced secondary minima due to eccentric orbits are detected.

into account according to the well-known nonlinearity formula for the Poisson distribution of impulses in the photon-counting flux. The dead time of our registration channel: photomultiplier + amplifier + pulse counter averaged 32.7 nanoseconds for the entire period of observations. This value has been carefully controlled, it remained stable within 5 percent. All observations were corrected for atmospheric extinction. More details of our observational method are described in [7], the instrumental system is presented in [6]. Since the number of nights at the Simeiz observatory of INASAN suitable for observations with a single-channel photometer is limited by weather conditions, we attempted to use CCD observations with standard and variable stars measured simultaneously. To attenuate the peak signal from such a bright star, images were defocused and exposures were shortened. The result was unsatisfactory. We failed to achieve the required accuracy better than 0.01 and the shape of the minima was distorted by systematic errors. The CCD observations in Stará Lesná observatory, Slovakia were much better. We used a small telescope with a $15-\mathrm{cm}$ aperture, so it was not necessary to attenuate the signal artificially. In addition, a larger field of view ensured the presence of a bright comparison star in one frame with a variable star. In the spring 2021 we obtained at the Simeiz observatory of INASAN only the incomplete observations in four minima during unsatisfactory weather conditions. But these observations turned out to be very important for determination of the parameters of the external orbit, in relation to each of the eclipsing systems.

BU CMi was also measured six times during observations of the SAI catalogue of bright stars [8]. We recalculated the ultraviolet $W$ values given in this catalogue into the standard $U$ system, and the instrumental values $B, V$ were corrected a little to correspond precisely to Johnson system. Two measurements were obtained during the weakening of the brightness, which enable us to fix the moment of the minimum farthest from the current epoch, and four measurements turned out to be located between the minima and were averaged. Data of the brightness of $\mathrm{BU} \mathrm{CMi}$ on the plateau and comparison star are given in Table 2.

Table 1. Observation log

\begin{tabular}{c|c|l|l}
\hline Year & $\begin{array}{c}\text { Number of observa- } \\
\text { tional nights }\end{array}$ & \multicolumn{1}{|c}{$\begin{array}{c}\text { Photometric } \\
\text { passband }\end{array}$} & \multicolumn{1}{|c}{ Telescope and receiving equipment } \\
\hline 2012 & 7 & $V$ & $15 \mathrm{~cm}$ Maksutov, Stará Lesná, Slovakia, CCD ST-10XME \\
2020 & 20 & $U B V$ & Zeiss-600, Simeiz, INASAN, PMT EMI 9789B \\
2021 & 4 & $V$ & Zeiss-600, Simeiz, INASAN, PMT EMI 9789B \\
\hline
\end{tabular}


Table 2. Stellar magnitudes of BU CMi on plateau and standard star in Johnson system

\begin{tabular}{c|c|r|r}
\hline Star & $V$ & \multicolumn{1}{|c}{$U-B$} & \multicolumn{1}{c}{$B-V$} \\
\hline BU CMi & 6.419 & -0.070 & -0.035 \\
& 0.002 & 0.006 & 0.001 \\
HD64963 & 8.228 & 0.044 & 0.061 \\
& 0.002 & 0.007 & 0.002 \\
\hline
\end{tabular}

Also, at our request, T. Pribulla and R. Komžik carried out spectroscopic observations of the system using the high-resolution echelle spectrograph mounted on $1.35-\mathrm{m}$ reflector at the Skalnaté Pleso observatory, Slovakia [9]. Obtained spectra confirmed that the system contains moving lines of four different stars, with the spectral types close to A0 and approximately of the same brightness. We used the spectra to construct the radial-velocity curves for all four components of the system BU CMi.

\section{INTERSTELLAR EXTINCTION AND TEMPERATURES OF THE COMPONENTS}

The star under investigation is at a distance of 250 parsecs from us and is located far enough from the Galactic equator, $b=18^{\circ}$, so we cannot expect large interstellar absorption. This is confirmed by the position of the star on the two-colour $U-B, B-V$ diagram. We do not show it here, but a similar one can be seen in Fig. 3 of our previous work [7]. The colour indices of the standard star HD64963 do not show any significant interstellar reddening, too. According to [10], zero interstellar absorption in the direction of BU CMi follows up to a distance of several kiloparsecs. The maps of interstellar reddening [11, 12] suggest for BU CMi interstellar extinction $E(B-V)=0.007 \pm$ 0.0006 . Since it is difficult to determine the interstellar extinction with the same accuracy from our twocolour $U-B, B-V$ diagram, we accepted for further analysis this value of extinction and corrected the star colour index from Table 2. Despite the fact that reddening is close to zero, its value in the range of the colour indices corresponds to a temperature difference of $140 \mathrm{~K}$ according to calibration tables [13, 14].

It is interesting to note that although some young elliptical systems, such as GG Ori [15], V944 Cep [16], V2544 Cyg [17], and V839 Cep [18] exhibit anomalously large absorption, BU CMi does not show any significant deviations in absorption.

\section{THE PHOTOMETRIC ANALYSIS OF THE SYSTEM}

In our studies, we solve the complex problem of determining the entire set of interconnected charac- teristics of a multiple system, and the problem is complicated by the fact that there are two eclipsing binaries and the brightness of all four stars is measured simultaneously. Thus, the number of free parameters is at least double. A number of natural conditions limit the range of possible parameters of the model, which facilitates its construction. These conditions are as follows: First, the combined flux of the system must match the colour indices given in Table 2. Second, Kepler's laws must be fulfilled for each of the two eclipsing pairs separately, and for the external orbit for both pairs together. There may be some deviations from these laws, because each of the systems cannot be considered closed, the stars themselves cannot be considered as material points, as evidenced by the apsidal motion and small brightness variations between minima, see Fig. 1. But as a first approximation, the laws should work. Third, the photometrically determined distance to all four stars should be the same. Fourth, the total brightness of all four stars is taken as unity. The last condition immediately implies that we will solve the LCs with a significant contribution of the third light. Since the depths of all four minima are approximately the same and close to 0.2 , and the power of all lines is also approximately the same, it can be expected that all four stars have a similar surface brightness or, equivalently, temperature. We also assume that the empirical mass-luminosity law is fulfilled.

The applied technique is described in detail in a number of our previous articles, such as [7, 19-22]. Here, we just note that the stellar disk is represented by an ideal circle when modeling, the brightness distribution over it is taken into account by concentric circles of different brightness, [23], in the model of the linear law of darkening to the edge, and the final solution is obtained by the differential corrections method. Edgedarkening coefficients were fixed from the theoretical models [24]. Within the framework of this model, there are no brightness variations between the minima. The analysis revealed that in very accurate TESS observations, see Fig. 1, such changes at the level of fractions of a percent exist, but within the errors of the determined parameters, they do not affect the solutions at the minima.

There are three independent photometric sets in our disposal. The first set is the MASCARA unfiltered observations [3]. We solved them under the assumption that the average wavelength of the observations corresponds to the Johnson $R$ band. The second set comprise our own $U B V$ observations and the third set the TESS observations [4], to which the Cousins Ic band was attributed. Each of the available observational sets was analyzed separately and the results were compared. We got approximately the same independent parameters, and the light of " $A$ " and " $B$ " components was equally divided in all solutions. This is an important point. At the beginning, we received the 

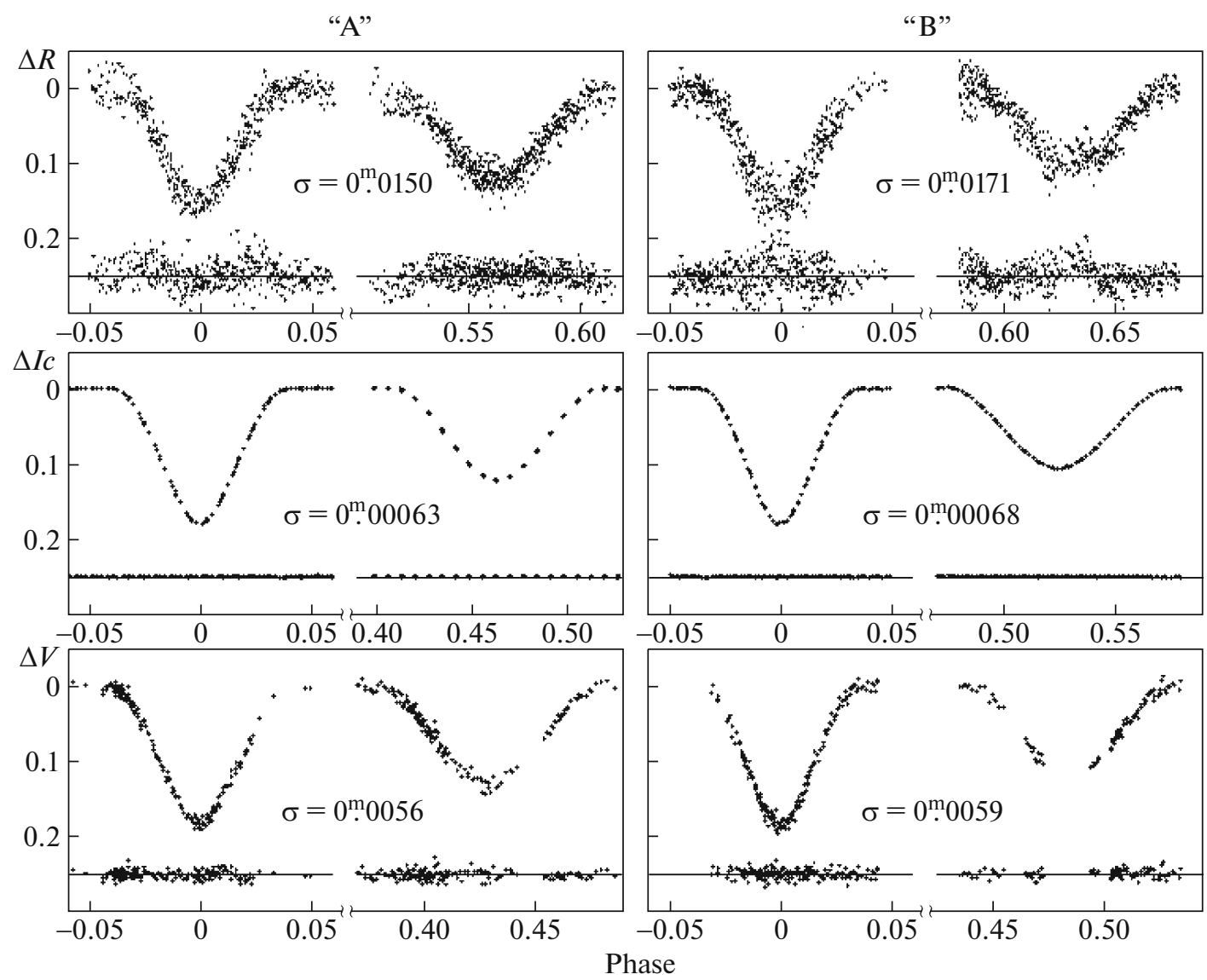

Fig. 2. Observations in minima. Left panels: component "A", right panels: component "B". The plateau level is assigned to zero. The $O-C$ residuals from concrete solution are presented under each minimum. The average error value is indicated. Observations are located in chronological order from top to bottom. Top row: MASCARA observations, middle row: TESS observations, lower row: our observations in the $V$ passband. A progressive decrease of the phases in the secondary minima caused by the fast apsidal movement is clearly seen.

same errors for our $V$ observations and TESS points, at the 0.006 level. But after a closer look at the residual deviations in TESS observations, it was found that by adding the small corrections to the time of observation at each date, the accuracy of the LC solution can be improved tenfold, from $0^{\mathrm{m}} .006$ to $0^{\mathrm{m}} .0006$. Later, having plotted on a large scale the graphs of the course of the $O-C$ residuals at the minima timings, we explained the introduction of these corrections by nutation, see the Section VI. The real weight of each individual observation increased by a factor of 100 . After correcting the TESS observations for this effect, we obtained the most accurate solution, which was taken as the final one. The approximation of our observations and MASCARA observations by this model does not worsen the scatter of points on the corresponding LCs. The results for all LCs are shown in Fig. 2. All these curves are approximated by the same parameters, except for the longitude of the periastron, which changes with time due to the rapid apsi- dal rotation. The parameters obtained from the solutions are presented in Table 3. The masses of the stars were estimated from photometric data using an indirect method, see [25]. The way in which we estimate the mass errors of the indirect method is described in [7]. The parameters found from the photometry served as the basis to disentangle the spectroscopic observations. Table 3 lists the masses already refined from the radial-velocity curves.

The obtained relative luminosities and temperatures, in accordance with the calibrations [13], correspond to the non-reddened colour index $B-V=-0^{\mathrm{m}} 031$. This value is larger by $0^{\mathrm{m}} .004$ than that measured in the catalogue [8], see Table 2. We believe that such a discrepancy is an objective assessment of both the accuracy of the calibrations [13] and the uncertainty of our solution, i.e., the match is satisfactory. 


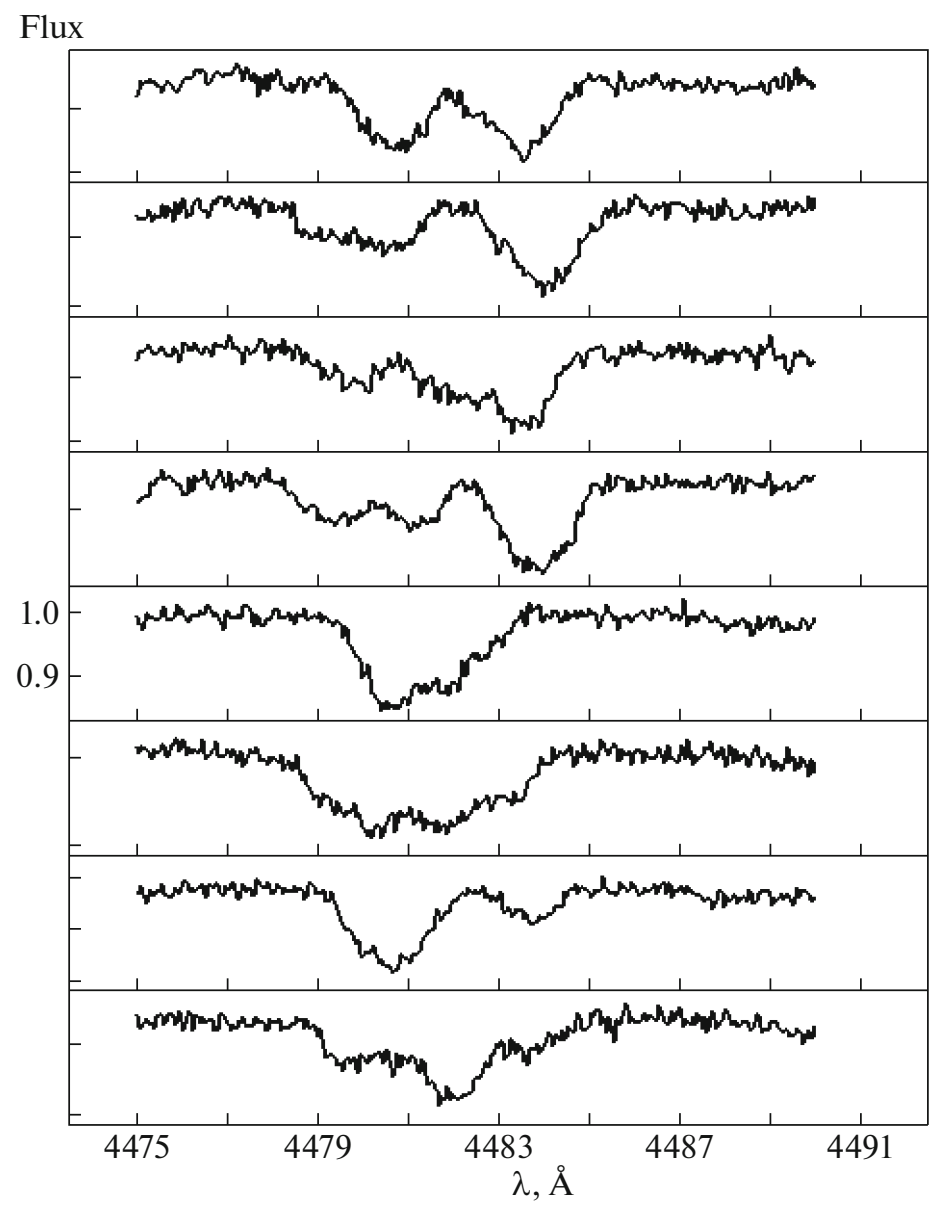

Fig. 3. The part of BU CMi spectrum near MgII (4481 $\AA$ ) line. The relative flux normalized to the continuum is plotted along the ordinate axis. The scale shown in one chart is the same for all charts. Every blend consists of at least two lines, so one can see the presence of moving lines from four components. These four lines have approximately the same intensity.

\section{THE SPECTRAL OBSERVATIONS AND THEIR ANALYSIS}

Disentangling the spectra in order to measure the radial velocities of the components turned out to be difficult task, because the line profile of each component was almost always in blend with the line profiles of other components. The line profiles of all four components were approximated by Gaussians of the same amplitude and width, and the best fit was sought between the resulting general approximation function and the observed spectrum. We used for spectral type A0 the most powerful optically thin lines $\mathrm{MgII}$ (4481 $\AA$ ), see Fig. 3. The results of the analysis are presented in Table 4 and in Figs. 4, 5. The significant scatter is due to the constant superposition of lines in the spectra. When solving the radial-velocity curves, the apsidal rotation was taken into account. The masses of the components are shown in Table 3 . These results are not final; work on the spectra is ongoing.

\section{THE APSIDAL ROTATION AND THE LIGHT TIME EFFECT IN MINIMA TIMINGS}

To study both effects, it is necessary to know the exact times of the minima. We have determined all possible timings from the MASCARA, TESS, and our observations. According to the conditions of the star's visibility above the horizon at the Simeiz observatory of INASAN, we were unable to observe any full eclipse. From the observed individual branches of the minima the average minima were constructed for our observations in 2020. Thus, we have obtained four average eclipse timings, one for each of the stars in the system. Another individual moment of minimum was obtained from our observations at Stará Lesná in 2012. The bibliographic search revealed only one published minimum timing [26]. One more timing we derived from the observations of the catalogue [8]. Although there are only two observational points at this minimum, the amplitude of the brightness attenuation indicates that they occurred at the time instant corresponding to the bottom of the minimum. Due to the 
Table 3. Absolute and relative parameters of BU CMi

\begin{tabular}{l|c|c}
\hline \multicolumn{1}{c|}{ Parameter } & Component “A” & Component "B" \\
\hline$r_{1}$ & $0.1712 \pm 0.005$ & $0.1461 \pm 0.005$ \\
$r_{2}$ & $0.1229 \pm 0.005$ & $0.1287 \pm 0.005$ \\
$i^{\circ}$ & $83.89 \pm 0.05$ & $83.40 \pm 0.05$ \\
$e$ & $0.204 \pm 0.007$ & $0.218 \pm 0.007$ \\
$\omega$, deg (JD 2458921) & $121.4 \pm 0.1$ & $96.05 \pm 0.1$ \\
$l_{1 v}$ & $0.336 \pm 0.001$ & $0.288 \pm 0.001$ \\
$l_{2 v}$ & $0.163 \pm 0.001$ & $0.213 \pm 0.001$ \\
$l_{1 b}$ & $0.335 \pm 0.002$ & $0.288 \pm 0.002$ \\
$l_{2 b}$ & $0.164 \pm 0.002$ & $0.213 \pm 0.002$ \\
$l_{1 u}$ & $0.343 \pm 0.003$ & $0.289 \pm 0.003$ \\
$l_{2 u}$ & $0.158 \pm 0.003$ & $0.210 \pm 0.003$ \\
$T_{1}$ & $10130 \pm 80 \mathrm{~K}$ & $10180 \pm 80 \mathrm{~K}$ \\
$T_{2}$ & $9740 \pm 80 \mathrm{~K}$ & $9890 \pm 80 \mathrm{~K}$ \\
Bolometric correction B.C. 1 & -0.277 & -0.286 \\
Bolometric correction B.C.2 & -0.196 & -0.227 \\
Mass, $M_{1}$ & $3.40 \pm 0.1 M_{\odot}$ & $3.29 \pm 0.1 M_{\odot}$ \\
Mass, $M_{2}$ & $3.11 \pm 0.1 M_{\odot}$ & $3.29 \pm 0.1 M_{\odot}$ \\
Radius, $R_{1}$ & $2.51 \pm 0.05 R_{\odot}$ & $2.31 \pm 0.05 R_{\odot}$ \\
Radius, $R_{2}$ & $1.804 \pm 0.05 R_{\odot}$ & $2.04 \pm 0.05 R_{\odot}$ \\
Free-fall acceleration, $\log g_{1}, \mathrm{~cm} / \mathrm{s}^{2}$ & $4.088 \pm 0.01$ & $4.148 \pm 0.01$ \\
Free-fall acceleration, $\log g_{2}, \mathrm{~cm} / \mathrm{s}^{2}$ & $4.338 \pm 0.01$ & $4.259 \pm 0.01$ \\
Major semiaxis $a, \mathrm{AU}$ & $16.1 \pm 0.1$ & $17.3 \pm 0.1$ \\
Photometric parallax, $\pi_{\mathrm{ph}}$ & 0.00407 " $\pm 0.00005^{\prime \prime}$ & 0.00407 " $\pm 0.00005^{\prime \prime}$ \\
\hline
\end{tabular}

Table 4. Radial velocities of BU CMi components

\begin{tabular}{c|c|c|c|c}
\hline HJD 2400000+ & $\begin{array}{c}\text { "A" primary, } \\
\mathrm{km} / \mathrm{s}\end{array}$ & $\begin{array}{c}\text { "A" secondary, } \\
\mathrm{km} / \mathrm{s}\end{array}$ & $\begin{array}{c}\text { "B” primary, } \\
\mathrm{km} / \mathrm{s}\end{array}$ & $\begin{array}{c}\text { "B" secondary, } \\
\mathrm{km} / \mathrm{s}\end{array}$ \\
\hline 59163.6152 & -30 & 40 & 110 & -100 \\
59164.6629 & 125 & -120 & -45 & 35 \\
59166.6279 & 25 & -45 & 80 & -50 \\
59177.6483 & -175 & 145 & 10 & 70 \\
59178.6138 & 5 & -45 & -85 & 115 \\
59179.6430 & 70 & -115 & 110 & -45 \\
59180.5556 & -180 & 145 & 105 & -75 \\
59185.6611 & 55 & -100 & -5 & 55 \\
59196.5649 & 65 & -110 & 155 & -70 \\
59197.5586 & -5 & -40 & -80 & 165 \\
59203.5284 & -25 & 25 & 130 & -30 \\
59216.4063 & -40 & 55 & 165 & -55 \\
59224.4806 & -145 & 150 & -25 & 100 \\
59226.4283 & 130 & -115 & 75 & -20 \\
59246.3586 & 60 & -95 & -95 & 165 \\
59246.3844 & 70 & -95 & -95 & 170 \\
\hline
\end{tabular}




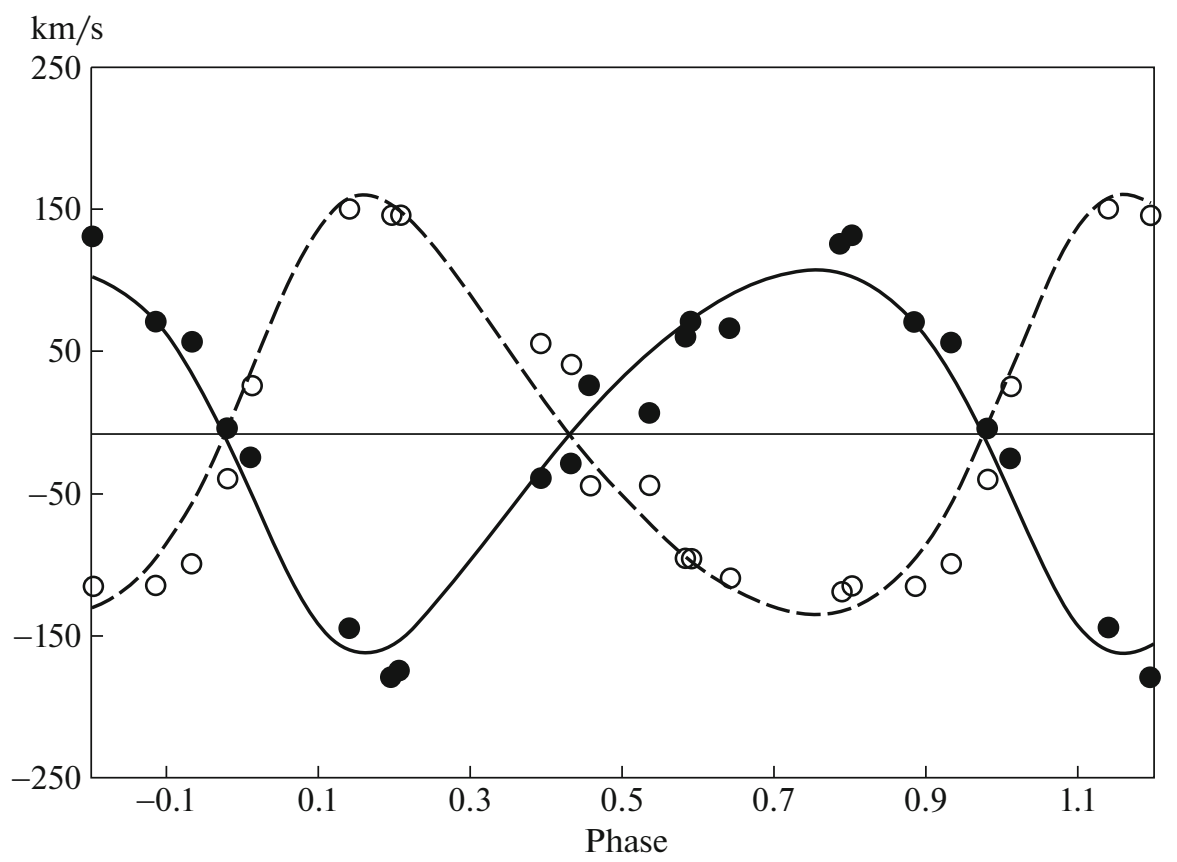

Fig. 4. Radial velocities of "A" star. Filled and open circles present the primary and the secondary component, respectively. The solid line corresponds to the solution of the radial velocity curve for the primary component and dashed line for the secondary one. The average error of a separate measurement is $13.3 \mathrm{~km} / \mathrm{s}$.

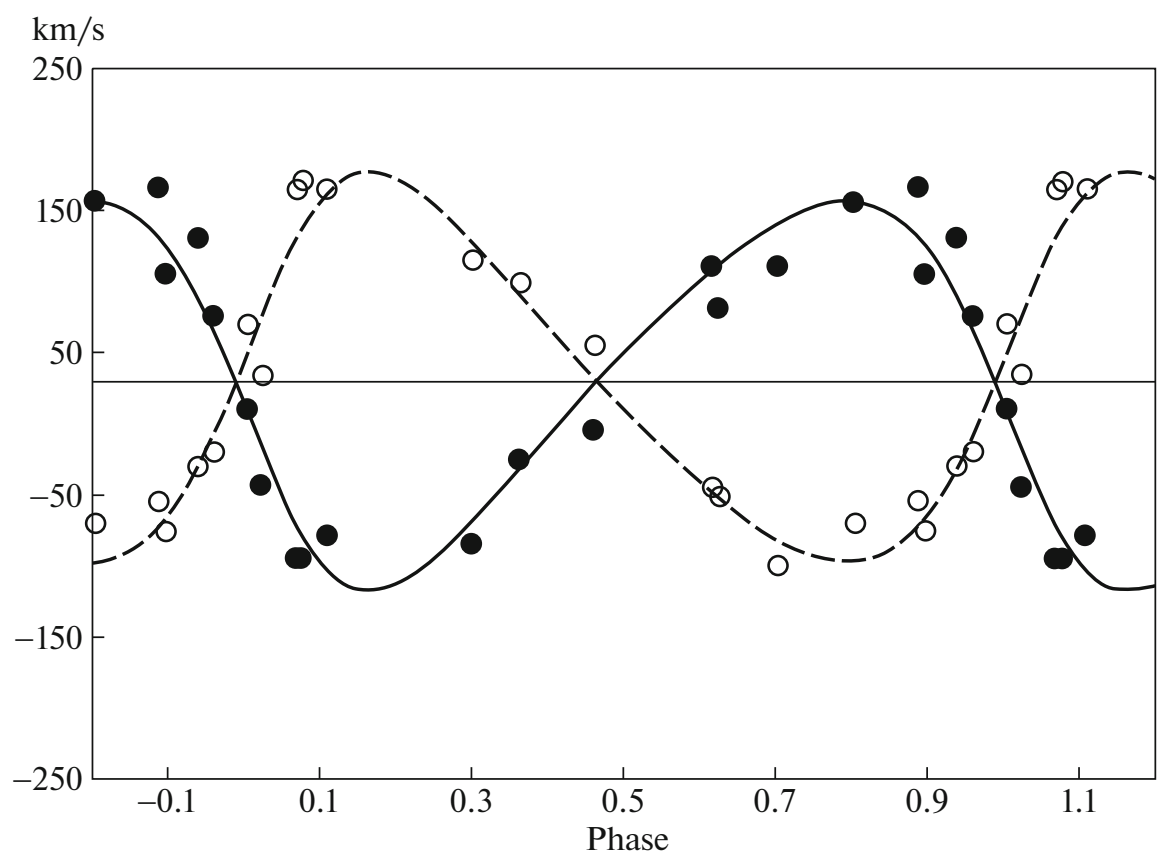

Fig. 5. Radial velocities of "B" star. Filled and open circles present the primary and the secondary component, respectively. The solid line corresponds to the solution of the radial velocity curve for the primary component and dashed line for the secondary one. The average error of a separate measurement is $15.5 \mathrm{~km} / \mathrm{s}$.

fact that the observations were obtained in the most distant time from the modern era it most accurately determines the period of revolution in the outer orbit.
And finally, from observations of the parts of the minima in 2021 using the established geometric model of the star, the four most recent minima timings were 


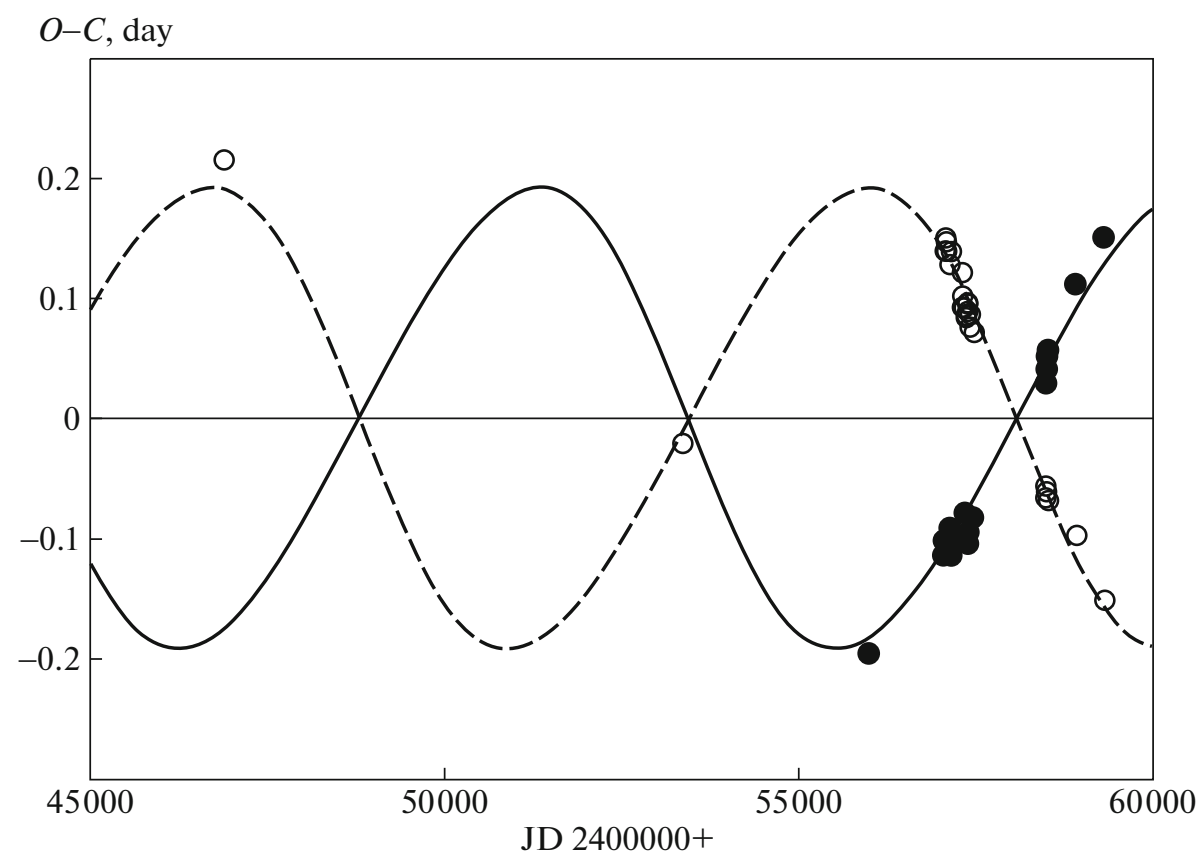

Fig. 6. The $O-C$ residuals calculated with the same period for the primary and the secondary minima for "A" component. The primary and the secondary minima are shown as open circles. The solid and dashed lines correspond to detected apsidal motion for the primary and the secondary minima, respectively.

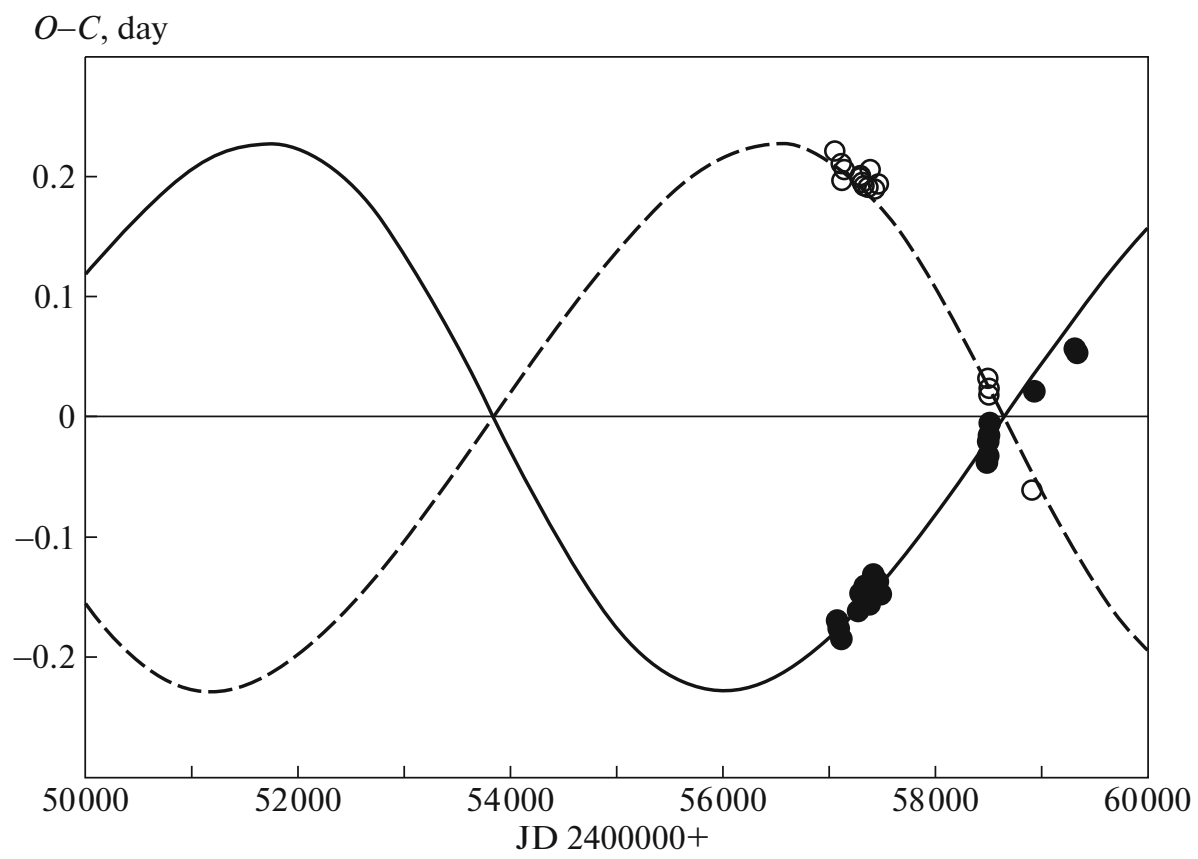

Fig. 7. The $O-C$ residuals calculated with the same period for the primary and the secondary minima for "B" component. The primary and the secondary minima are shown as open circles. The solid and dashed lines correspond to detected apsidal motion for the primary and the secondary minima, respectively.

obtained. For each of the available timings it was determined which component of the quadruple system is eclipsed, the blending minima were discarded. All obtained data are presented in Tables 5, 6, 7, 8.
The largest contribution to the deviation of the course of the minima timings from the linear formula is made by the apsidal rotation. The amplitude of the effect reaches 0.2 days. The next in importance is the 

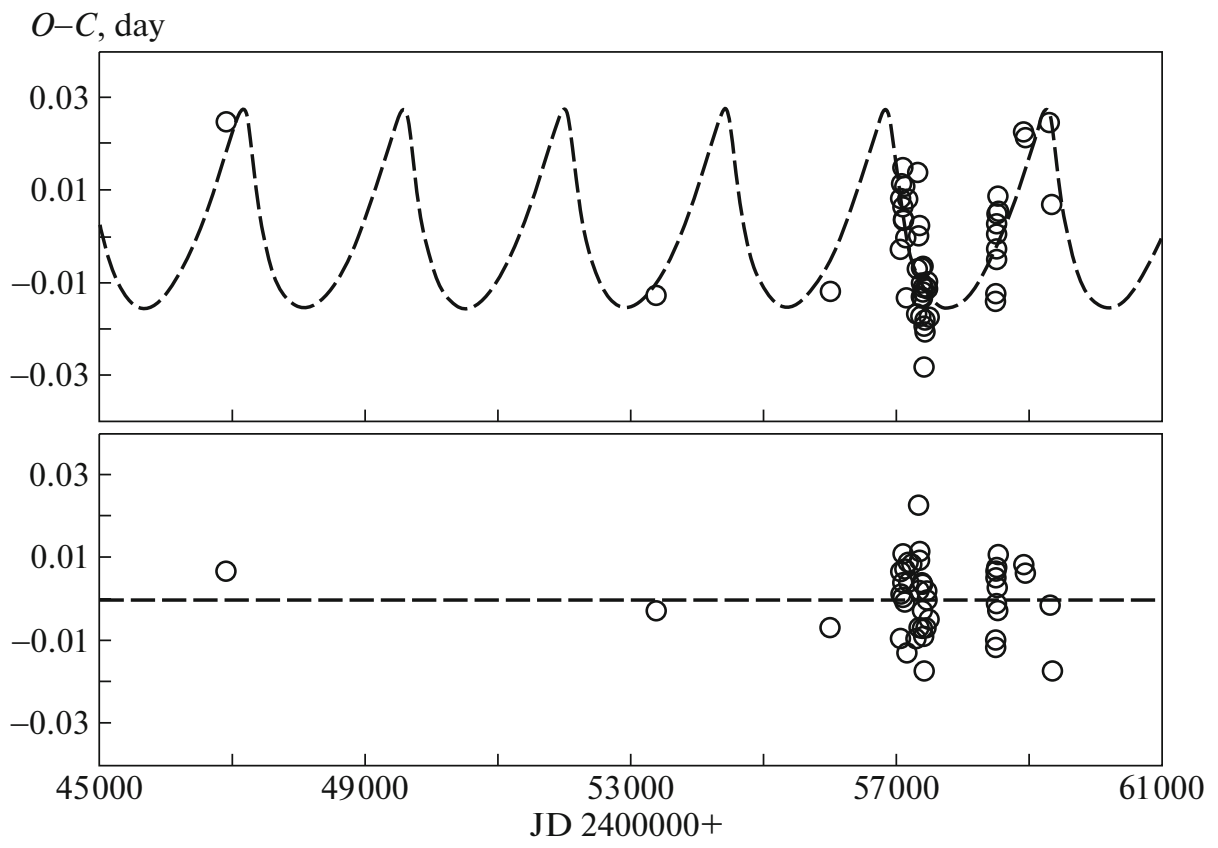

Fig. 8. Top panel: The $O-C$ graph after subtracting the apsidal movement for the "A" component. The dashed line corresponds to the parameters of the external orbit of the "A" component around the center of gravity of "A" + "B" system. Lower panel: residual deviations after subtracting the apsidal and orbital movements. The residual scatter is associated with the orbit nutation in the periastron longitude.

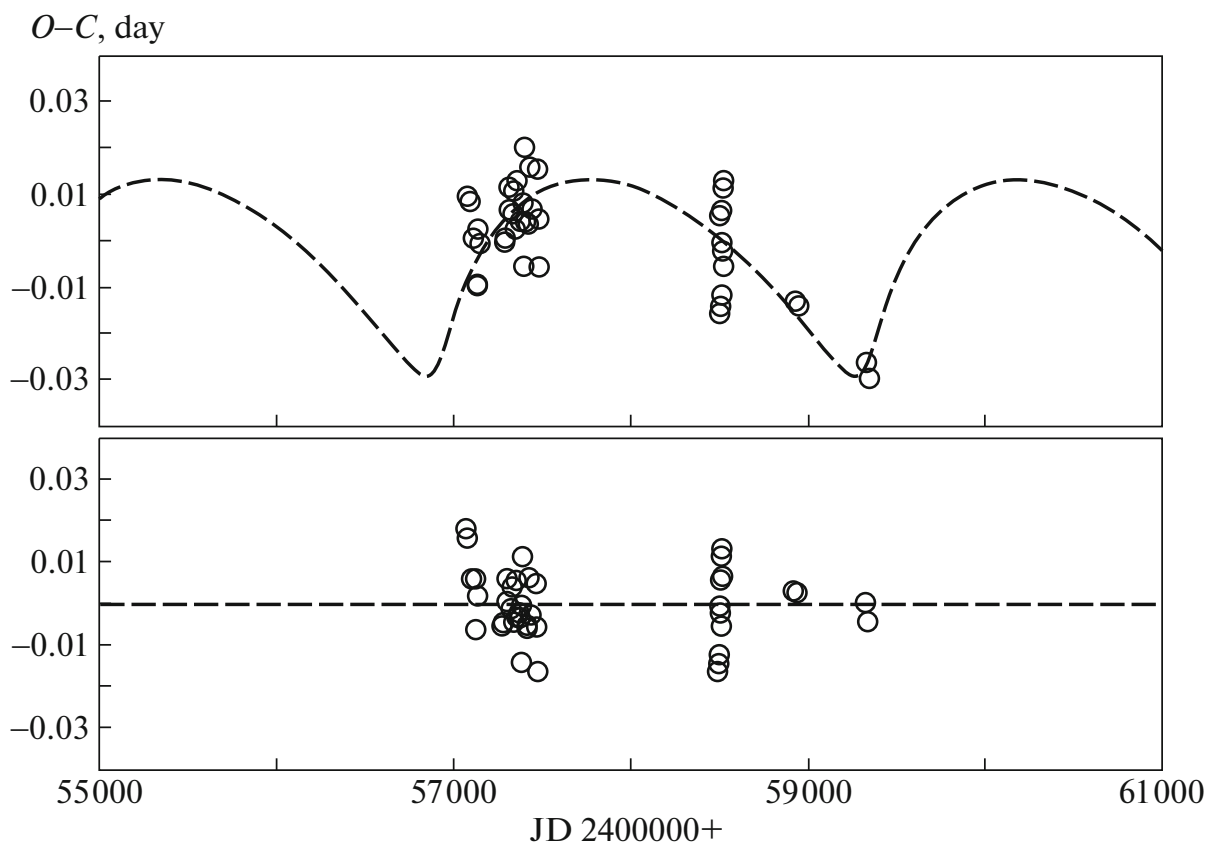

Fig. 9. Top panel: The $O-C$ graph after subtracting the apsidal movement for the " $\mathrm{B}$ " component. The dashed line corresponds to the parameters of the external orbit of the "B" component around the center of gravity of " $A$ " + " $B$ " system. Lower panel: residual deviations after subtracting the apsidal and orbital movements. The residual scatter is associated with the orbit nutation in the periastron longitude. 
Table 5. The primary minima timings of " $A$ " component

\begin{tabular}{l|l|l|l|l}
\hline \multicolumn{1}{c|}{$\begin{array}{c}\text { HJD } \\
(2400000+)\end{array}$} & $\begin{array}{c}(O-C)_{1}, \\
\text { days }\end{array}$ & $\begin{array}{c}(O-C)_{2}, \\
\text { days }\end{array}$ & $\begin{array}{c}(O-C)_{3}, \\
\text { days }\end{array}$ & Note \\
\hline 55993.22965 & -0.19522 & -0.01182 & -0.00674 & 3 \\
57057.439 & -0.101 & +0.008 & +0.001 & 4 \\
57060.367 & -0.112 & -0.003 & -0.010 & 4 \\
57098.591 & -0.103 & +0.003 & -0.000 & 4 \\
57107.419 & -0.099 & +0.006 & +0.004 & 4 \\
57110.357 & -0.095 & +0.010 & +0.007 & 4 \\
57151.492 & -0.114 & -0.013 & -0.013 & 4 \\
57160.332 & -0.092 & +0.008 & +0.009 & 4 \\
57286.721 & -0.104 & -0.017 & -0.009 & 4 \\
57336.715 & -0.082 & +0.000 & +0.009 & 4 \\
57342.596 & -0.080 & +0.002 & +0.011 & 4 \\
57380.802 & -0.088 & -0.010 & +0.001 & 4 \\
57386.674 & -0.095 & -0.018 & -0.007 & 4 \\
57389.618 & -0.090 & -0.013 & -0.003 & 4 \\
57392.552 & -0.096 & -0.020 & -0.009 & 4 \\
57395.494 & -0.094 & -0.018 & -0.007 & 4 \\
57398.423 & -0.104 & -0.028 & -0.017 & 4 \\
57436.658 & -0.083 & -0.011 & +0.001 & 4 \\
58492.06999 & +0.03187 & -0.01361 & -0.00995 & 5 \\
58495.00849 & +0.03083 & -0.01398 & -0.01142 & 5 \\
58497.94830 & +0.03109 & -0.01403 & -0.01156 & 5 \\
58506.77686 & +0.04101 & -0.00507 & -0.00289 & 5 \\
58509.72214 & +0.04675 & +0.00034 & +0.00242 & 5 \\
58512.66719 & +0.05226 & +0.00553 & +0.00752 & 5 \\
58515.61030 & +0.05582 & +0.00877 & +0.01066 & 5 \\
58921.32411 & +0.11242 & +0.02265 & +0.00838 & 6 \\
59312.3220 & +0.1508 & +0.0242 & -0.0015 & 6 \\
\hline & & & &
\end{tabular}

Roemer light-time effect (LITE) with the amplitude of 0.022 days due to the change in the distance to the stars "A" and "B" when they move in a common orbit. Then there are slightly smaller nutation fluctuations with an amplitude of about 0.015 days. The problem was solved using the programs specially developed for this case taking into account our experience in searching for invisible satellites in eclipsing systems [25, 2730]. The search for a solution was carried out by the method of successive approximations. Both the parameters of the apsidal rotation and the orbit of the third body were searched simultaneously. In this case, both external orbits for components " $A$ " and " $B$ " had to be identical and differ only in the longitude of the periastron (by 180 degrees) and the amplitudes of the LITE - due to the difference in the masses of both systems. It was the observations of the " $A$ " component that made it possible to fix the parameters of the outer orbit most reliably, since for it, the number of observed
Table 6. The secondary minima timings of " $A$ " component

\begin{tabular}{l|l|l|l|l}
\hline $\begin{array}{c}\text { HJD } \\
(2400000+)\end{array}$ & $\begin{array}{c}(\mathrm{O}-\mathrm{C})_{1}, \\
\text { days }\end{array}$ & $\begin{array}{c}(\mathrm{O}-\mathrm{C})_{2}, \\
\text { days }\end{array}$ & $\begin{array}{c}(\mathrm{O}-\mathrm{C})_{3}, \\
\text { days }\end{array}$ & Note \\
\hline 46897.220 & +0.215 & +0.025 & +0.007 & 1 \\
53378.6805 & -0.0209 & -0.0128 & -0.0028 & 2 \\
57085.615 & +0.147 & +0.011 & +0.007 & 4 \\
57091.497 & +0.150 & +0.015 & +0.011 & 4 \\
57094.425 & +0.139 & +0.004 & +0.002 & 4 \\
57097.371 & +0.145 & +0.010 & +0.007 & 4 \\
57144.388 & +0.129 & -0.000 & -0.000 & 4 \\
57147.335 & +0.137 & +0.008 & +0.008 & 4 \\
57323.673 & +0.102 & -0.007 & +0.002 & 4 \\
57329.542 & +0.092 & -0.016 & -0.007 & 4 \\
57332.511 & +0.122 & +0.014 & +0.023 & 4 \\
57367.750 & +0.086 & -0.017 & -0.007 & 4 \\
57370.690 & +0.086 & -0.017 & -0.006 & 4 \\
57373.628 & +0.085 & -0.018 & -0.007 & 4 \\
57376.578 & +0.096 & -0.007 & +0.004 & 4 \\
57379.511 & +0.089 & -0.013 & -0.003 & 4 \\
57382.456 & +0.095 & -0.007 & +0.004 & 4 \\
57385.393 & +0.091 & -0.010 & +0.001 & 4 \\
57423.591 & +0.076 & -0.021 & -0.009 & 4 \\
57426.541 & +0.086 & -0.010 & +0.002 & 4 \\
57429.478 & +0.084 & -0.012 & +0.000 & 4 \\
57432.411 & +0.077 & -0.018 & -0.006 & 4 \\
57479.438 & +0.072 & -0.017 & -0.004 & 4 \\
58493.45422 & -0.05545 & +0.00465 & +0.00726 & 5 \\
58496.39330 & -0.05591 & +0.00461 & +0.00713 & 5 \\
58499.33059 & -0.05816 & +0.00277 & +0.00519 & 5 \\
58505.20707 & -0.06078 & +0.00100 & +0.00323 & 5 \\
58508.14223 & -0.06516 & -0.00296 & -0.00082 & 5 \\
58511.07922 & -0.06772 & -0.00510 & -0.00306 & 5 \\
58934.34435 & -0.09707 & +0.02100 & +0.00613 & 6 \\
59331.1291 & -0.1509 & +0.0068 & -0.0172 & 6 \\
\hline & & & &
\end{tabular}

minima is maximal and they are distributed over a significant time interval of 34 years. For the apsidal rotation of both eclipsing systems, the parameters presented in Table 9 were obtained.

After deduction of the apsidal rotation, the graphs of which are shown in Figs. 6 and 7, the parameters of the third body's orbit that most closely match the observed course of the $O-C$ residuals (see Figs. 8 and 9), are as follows:

$$
\begin{gathered}
P_{3}=2420 \pm 40 \text { day, or } 6.62 \text { years, } \\
T_{0}=\text { J.D. } 2454490 \pm 20, \\
(A \sin i)_{\mathrm{A}}=4.21 \pm 0.08 \mathrm{AU},
\end{gathered}
$$


Table 7. The primary minima timings of "B" component

\begin{tabular}{l|l|l|l|l}
\hline \multicolumn{1}{c|}{ HJD } & $(O-C)_{1}$, & \multicolumn{1}{c}{$(O-C)_{2}}$, & $(O-C)_{3}$, \\
days & days & Note \\
\hline 57076.560 & -0.170 & +0.009 & +0.016 & 4 \\
57099.390 & -0.176 & +0.001 & +0.006 & 4 \\
57125.480 & -0.184 & -0.010 & -0.006 & 4 \\
57278.830 & -0.161 & -0.000 & -0.005 & 4 \\
57301.680 & -0.147 & +0.012 & +0.006 & 4 \\
57327.780 & -0.145 & +0.011 & +0.004 & 4 \\
57350.620 & -0.141 & +0.013 & +0.005 & 4 \\
57386.490 & -0.156 & -0.006 & -0.014 & 4 \\
57399.550 & -0.145 & +0.004 & -0.005 & 4 \\
57412.600 & -0.144 & +0.004 & -0.006 & 4 \\
57422.400 & -0.131 & +0.016 & +0.006 & 4 \\
57461.540 & -0.138 & +0.005 & -0.006 & 4 \\
57474.580 & -0.147 & -0.006 & -0.016 & 4 \\
58492.51817 & -0.03701 & -0.01660 & -0.01620 & 5 \\
58495.78232 & -0.03513 & -0.01414 & -0.01464 & 5 \\
58499.04752 & -0.03220 & -0.01162 & -0.01202 & 5 \\
58505.58412 & -0.02014 & -0.00039 & -0.00058 & 5 \\
58508.85361 & -0.01292 & +0.00642 & +0.00634 & 5 \\
58512.12118 & -0.00762 & +0.01130 & +0.01132 & 5 \\
58515.38576 & -0.00531 & +0.01320 & +0.01333 & 5 \\
58936.24470 & +0.02080 & -0.01410 & +0.00261 & 6 \\
59321.22804 & +0.05628 & -0.02604 & +0.00051 & 6 \\
59334.27505 & +0.05421 & -0.02967 & -0.00437 & 6 \\
\hline
\end{tabular}

$$
\begin{gathered}
(A \sin i)_{\mathrm{B}}=4.14 \pm 0.08 \mathrm{AU}, \\
e=0.70 \pm 0.03 \\
\omega_{\mathrm{A}}=132^{\circ} \pm 3^{\circ} \\
\omega_{\mathrm{B}}=312^{\circ} \pm 3^{\circ} \\
f\left(M_{3}\right)_{\mathrm{A}}=1.6877 \pm 0.0005 M_{\odot}, \\
f\left(M_{3}\right)_{\mathrm{B}}=1.6086 \pm 0.0005 M_{\odot} .
\end{gathered}
$$

The errors are underestimated, they correspond to the specific model, but there could exist several models which correspond to their own local minima. So the parameters presented will not necessarily be final. The period of the outer orbit is not fully covered by observations yet. Our study of the entire area of feasible solutions showed that there is a set of parameters which differ from each other beyond the errors presented. The real period of the outer orbit falls in 5.97.7 years interval. We can also estimate that the ratio of the masses of the system " $\mathrm{A}$ " to system " $\mathrm{B}$ " is approximately 1.0. This result is in good agreement with photometric solutions, in which the luminosities of components "A" and "B" are equally divided. Application of Kepler's third law to the obtained parameters of
Table 8. The secondary minima timings of " $\mathrm{B}$ " component

\begin{tabular}{l|l|l|l|l}
\hline $\begin{array}{c}\text { HJD } \\
(2400000+)\end{array}$ & $\begin{array}{c}(\mathrm{O}-\mathrm{C})_{1}, \\
\text { days }\end{array}$ & $\begin{array}{c}(\mathrm{O}-\mathrm{C})_{2}, \\
\text { days }\end{array}$ & $\begin{array}{c}(\mathrm{O}-\mathrm{C})_{3}, \\
\text { days }\end{array}$ & Note \\
\hline 57065.570 & +0.221 & +0.010 & +0.018 & 4 \\
57124.280 & +0.210 & +0.003 & +0.006 & 4 \\
57127.530 & +0.198 & -0.009 & -0.006 & 4 \\
57137.325 & +0.206 & -0.001 & +0.002 & 4 \\
57280.855 & +0.196 & +0.000 & -0.005 & 4 \\
57303.695 & +0.200 & +0.007 & +0.001 & 4 \\
57329.790 & +0.197 & +0.006 & -0.001 & 4 \\
57342.835 & +0.193 & +0.003 & -0.005 & 4 \\
57365.670 & +0.192 & +0.004 & -0.004 & 4 \\
57375.460 & +0.195 & +0.008 & -0.000 & 4 \\
57388.520 & +0.206 & +0.020 & +0.012 & 4 \\
57427.650 & +0.189 & +0.007 & -0.003 & 4 \\
57463.540 & +0.194 & +0.015 & +0.005 & 4 \\
58494.25583 & +0.03357 & +0.00528 & +0.00573 & 5 \\
58497.51828 & +0.03275 & +0.00603 & +0.00558 & 5 \\
58507.29522 & +0.02288 & -0.00213 & -0.00226 & 5 \\
58510.55370 & +0.01909 & -0.00535 & -0.00538 & 5 \\
58921.52009 & -0.06054 & -0.01305 & +0.00300 & 6 \\
\hline
\end{tabular}

Footnotes to Tables 5, 6, 7, 8: The errors of minima times approximately correspond to 5 units of the last significant digit. $(\mathrm{O}-\mathrm{C})_{1}-$ deviation from the linear formula. $(\mathrm{O}-\mathrm{C})_{2}$-residuals after the deduction of the apsidal movement. $(\mathrm{O}-\mathrm{C})_{3}$-residuals after the deduction of the apsidal movement and the light-time effect.

Notes: (1) - data obtained from the SAI catalogue [8], (2) [25], (3) CCD observations, Stará Lesná, Slovakia, (4) MASCARA observations [3], (5) TESS observations [4], (6) Observations with a photomultiplier, INASAN Simeiz Observatory.

Table 9. Elements of the apsidal motion of BU CMi components. Values of eccentricity are fixed from the photometric solutions

\begin{tabular}{l|l|l}
\hline \multicolumn{1}{c|}{ Parameter } & \multicolumn{1}{|c}{ “A” } & \multicolumn{1}{c}{ "B" } \\
\hline T0 [HJD] & 2458092.260 & 2457076.730 \\
$P_{\mathrm{A}}$ [days] & $2.939545(2)$ & $3.26227(1)$ \\
$e$ & 0.20425 & 0.2181 \\
$\omega[$ deg $/$ year] & $14.2(5)$ & $13.7(5)$ \\
$\omega_{0}[$ deg] & 90 & 30.65 \\
$U$ [year] & $25.4(1)$ & $26.3(1)$ \\
\hline
\end{tabular}

external orbit gives the total value of the mass of the entire system as $M(\sin i)^{3}=13.3 M_{\odot}$. The solutions to the radial-velocity curves assume $M=13.1 M_{\odot}$ (the inclination angles of the orbits are known with high accuracy from the photometric solutions). Hence, we conclude that $\sin i \approx 1$, i.e., the angle of inclination of the outer orbit is close to the normal and corresponds 

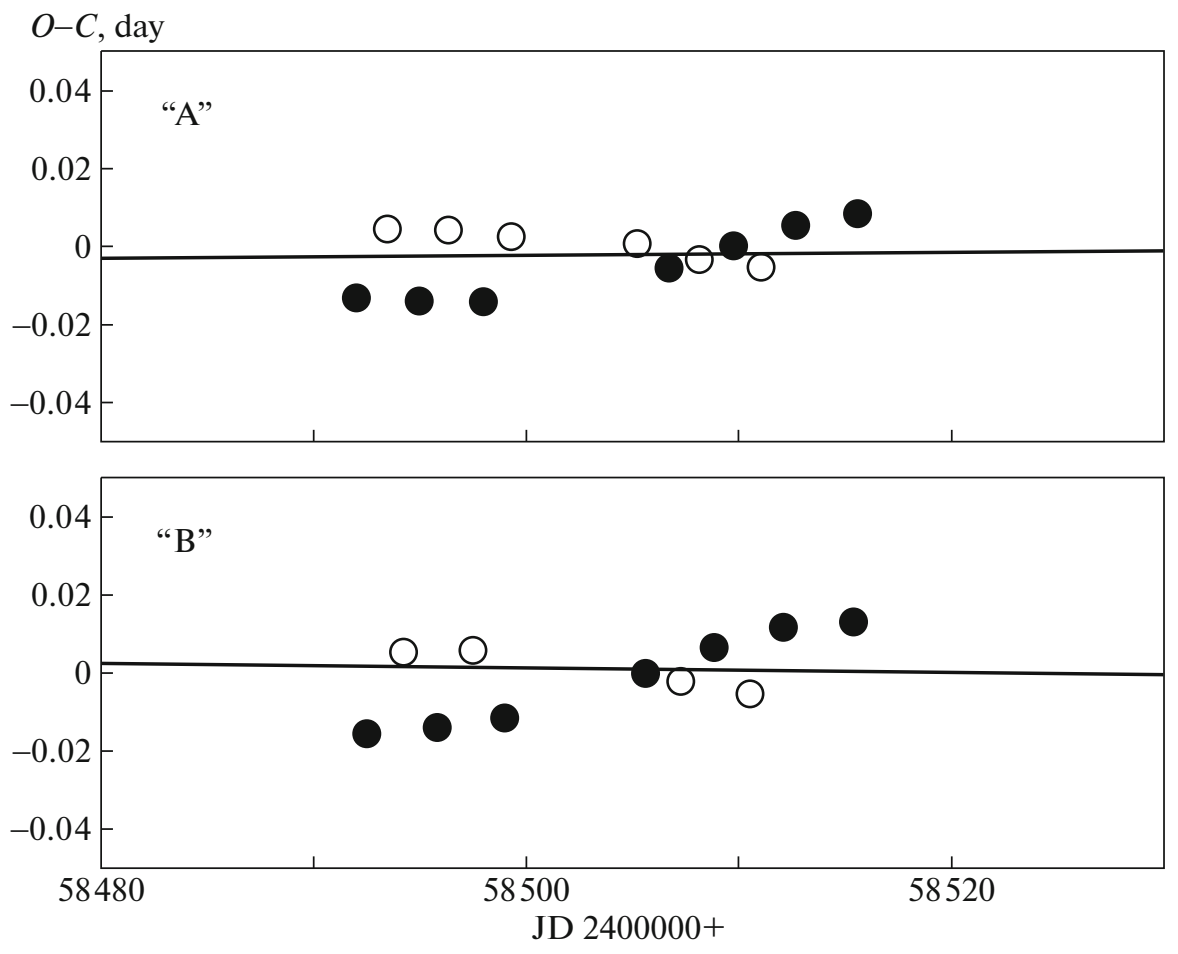

Fig. 10. Figures 8, 9 on an enlarged scale for the TESS observations. Filled and open circles present the primary and the secondary minima, respectively. The solid oblique line corresponds to the Roemer effect due to the orbital motion of the " $A$ " and " $B$ " components. The orbit nutation can explain observed oscillations in antiphase for the primary and the secondary minima of the components "A" (upper panel) and "B" (lower panel).

to the angles of inclination of each of the eclipsing systems to the line of sight. All three orbits are most likely located in the same plane. But for final conclusions about the external orbit of the system further observations are required. We cannot rule out that the above set of parameters of the outer orbit corresponds to one of the local minima.

A large-scale $O-C$ plots for individual TESS timings is shown in Fig. 10. It can be seen that the primary and the secondary minima for each of the " $A$ " and " $B$ " components slant in antiphase. Such a picture can be obtained if we assume that the elliptical orbits of each of the " $A$ " and " $B$ " components wobble within one degree in the longitude of the periastron. We estimated the periods of such wobbling at 60 days, and for both eclipsing stars they are approximately the same. It turns out that in addition to the usual effects observed in double elliptical systems, such as apsidal motion and the LITE due to the influence of the third body, nutation can also be present.

We do not give here the calculations of the theoretically expected apsidal rotation. For such orbits and masses of the components, it is obviously smaller than the measured values. The reasons for the rapid apsidal rotation must be related to the mutual influence of both systems on each other. Synchronization is clearly observed in the system in the orbital, nutation and apsidal motions of the components " $A$ " and " $B$ ": the periods of the eclipsing pairs are $P_{\mathrm{A}} / P_{\mathrm{B}}=0.9$, the periods of nutation are approximately equal, the apsidal periods are also the same. The orbital planes of both eclipsing systems are collinear. It can be concluded that this multiple system has already reached an equilibrium state and is stable.

\section{CONCLUSIONS}

We have revealed the nature of the unusual quadruple eclipsing system BU CMi, consisting of two eclipsing binaries. The periods of both binaries are tied up by a resonance of 9/10. A fast apsidal rotation is observed in each binary. We built a preliminary orbit in which both binaries rotate around a common center of mass. We found the physical characteristics of all four stars: temperatures, sizes, masses. All stars appear to be very young, aged up to 200 million years. A preliminary comparison of the obtained parameters with theoretical models indicates that the chemical composition of the stars does not correspond to the solar one, and the secondary components are younger than the primary ones. This fact is not surprising in the case of young stars. Under the condition of common origin, less massive stars should set on the ZAMS later than more massive ones. Further intensive photometric and spectral observations are needed to refine the parameters of the mutual orbit and to study nutation. We estimate the angular distance between the " $\mathrm{A}$ " and " $\mathrm{B}$ " 
components at the current moment to be about $0.01^{\prime \prime}$. So, it is possible to directly measure the components of a binary star using speckle interferometry. BU CMi system is very interesting from the point of view of stellar formation and evolution theory, as well as for studying the dynamics of close multiple systems.

\section{ACKNOWLEDGMENTS}

The observations were fulfilled with the $60-\mathrm{cm}$ reflector of the Simeiz observatory of INASAN. This work has used the SIMBAD database of the Strasbourg Center for Astronomical Data (France) and the bibliographic reference service of the ADS (NASA, USA). This document includes data collected by the TESS mission. Funding for the TESS mission is provided by NASA's Science Missions Office. We are grateful to the staff members of the Astronomical Institute of the Slovak Academy of Sciences T. Pribulla and R. Komžik for the spectroscopic observations carried out at our request. We are also grateful to the anonymous referee, whose valuable comments led to a significant improvement of the article.

\section{FUNDING}

This work was supported by a scholarship of Slovak Academic Information Agency SAIA (ASK, IMV), RFBR grants 11-02-01213a and 18-502-12025 (IMV), the Program of development of Lomonosov Moscow State University "Leading Scientific School Physics of stars, relativistic objects and galaxies" (IMV), and the Slovak Research and Development Agency under the contract no. APVV-15-0458 (DC) and grant VEGA 2/0031/18 (DC).

\section{OPEN ACCESS}

This article is licensed under a Creative Commons Attribution 4.0 International License, which permits use, sharing, adaptation, distribution and reproduction in any medium or format, as long as you give appropriate credit to the original author(s) and the source, provide a link to the Creative Commons license, and indicate if changes were made. The images or other third party material in this article are included in the article's Creative Commons license, unless indicated otherwise in a credit line to the material. If material is not included in the article's Creative Commons license and your intended use is not permitted by statutory regulation or exceeds the permitted use, you will need to obtain permission directly from the copyright holder. To view a copy of this license, visit http://creativecommons.org/licenses/by/4.0/.

\section{REFERENCES}

1. N. N. Samus, E. V. Kazarovets, O. V. Durlevich, N. N. Kireeva, and E. N. Pastukhova, Astron. Rep. 61, 80 (2017).

2. I. M. Volkov and N. S. Volkova, Astron. Rep. 53, 136 (2009).

3. O. Burggraaff, G. J. J. Talens, J. Spronck, et al., Astron. Astrophys. 617, A32 (2018).
4. K. G. Stassun, R. J. Oelkers, M. Paegert, et al., Astron. J. 158, 158 (2019).

5. G. Poymanski, Acta Astron. 52, 397 (2002).

6. I. M. Volkov and N. S. Volkova, Astron. Astrophys. Trans. 26, 129 (2007).

7. I. M. Volkov, A. S. Kravtsova, and D. Chochol, Astron. Rep. 65, 184 (2021).

8. V. G. Kornilov, I. M. Volkov, A. I. Zakharov, et al., WBVR Catalogue of Bright Northern Stars (Mosk. Gos. Univ., Moscow, 1991) [in Russian].

9. T. Döhring, T. Pribulla, R. Komžik, M. Mann, P. Sivanič, and M. Stollenwerk, Contrib. Astron. Obs. Skalnaté Pleso 49, 154 (2019).

10. G. M. Green, E. F. Schlafly, D. P. Finkbeiner, H.-W. Rix, et al., Astrophys. J. 810, 25 (2015).

11. E. F. Schlafly and D. P. Finkbeiner, Astrophys. J. 737, 103 (2011).

12. D. J. Schlegel, D. P. Finkbeiner and M. Davis, Astrophys. J. 500, 525 (1998).

13. P. J. Flower, Astrophys. J. 469, 355 (1996).

14. D. M. Popper, Ann. Rev. Astron. Astrophys. 18, 115 (1980).

15. I. M. Volkov and Kh. F. Khaliullin, Astron. Rep. 46, 747 (2002).

16. I. Volkov, D. Chochol, and L. Bagaev, in Living Together: Planets, Host Stars and Binaries, Proc. Conference, Sept. 8-12, 2014, Litomysl, Czech Republic, Ed. by S. M. Rucinski, G. Torres, and M. Zejda, ASP Conf. Ser. 496, 266 (2015).

17. I. Volkov, D. Chochol, and L. Bagaev, in Proceedings of the Conference on the Impact of Binaries on Stellar Evolution, ESO Garching, Germany, July 3-7, 2017.

18. I. M. Volkov, L. A. Bagaev, A. S. Kravtsova, and D. Chochol, Contrib. Astron. Obs. Skalnaté Pleso 49, 434 (2019).

19. I. M. Volkov, D. Chochol, and A. S. Kravtsova, Astron. Rep. 61, 440 (2017).

20. L. A. Bagaev, I. M. Volkov, and I. V. Nikolenko, Astron. Rep. 62, 664 (2018).

21. A. S. Kravtsova, I. M. Volkov, and D. Chochol, Astron. Rep. 63, 495 (2019).

22. I. M. Volkov and A. S. Kravtsova, Astron. Rep. 64, 211 (2020).

23. Kh. F. Khaliullin and A. I. Khaliullina, Sov. Astron. 28, 228 (1984).

24. R. A. Wade and S. M. Rucinski, Astron. Astrophys. Suppl. Ser. 60, 471 (1985).

25. I. M. Volkov, D. Chochol, J. Grygar, M. Mašek, and J. Juryšek, Contrib. Astron. Obs. Skalnaté Pleso 47, 29 (2017).

26. W. Ogloza, W. Niewiadomski, A. Barnacka, M. Biskup, K. Malek, and M. Sokolowski, Inform. Bull. Variable Stars 5843, 1 (2008).

27. I. M. Volkov, D. Chochol, N. S. Volkova, and I. V. Nikolenko, Proc. IAU 282, 89 (2012).

28. I. M. Volkov and N. S. Volkova, ASP Conf. Ser. 435, 323 (2010).

29. N. Volkova and I. Volkov, IBVS Inform. Bull. Variable Stars 5976, 1 (2011).

30. I. M. Volkov, ASP Conf. Ser. 496, 109 (2015). 